ARTICLE

Received 25 Feb 2014 | Accepted 30 Sep 2014 | Published 18 Nov 2014

DOI: $10.1038 /$ ncomms6421 OPEN

\title{
Structure and function of a spectrin-like regulator of bacterial cytokinesis
}

Robert M. Cleverley ${ }^{1}$, Jeffrey R. Barrett ${ }^{2}$, Arnaud Baslé ${ }^{1}$, Nhat Khai Bui ${ }^{1}{ }^{\dagger}$, Lorraine Hewitt ${ }^{1}$, Alexandra Solovyova $^{3}$, Zhi-Qiang Xu², Richard A. Daniel ${ }^{1}$, Nicholas E. Dixon², Elizabeth J. Harry ${ }^{4}$, Aaron J. Oakley², Waldemar Vollmer ${ }^{1}$ \& Richard J. Lewis ${ }^{1}$

Bacterial cell division is facilitated by a molecular machine-the divisome-that assembles at mid-cell in dividing cells. The formation of the cytokinetic Z-ring by the tubulin homologue FtsZ is regulated by several factors, including the divisome component EzrA. Here we describe the structure of the 60-kDa cytoplasmic domain of EzrA, which comprises five linear repeats of an unusual triple helical bundle. The EzrA structure is bent into a semicircle, providing the protein with the potential to interact at both $\mathrm{N}$ - and $\mathrm{C}$-termini with adjacent membrane-bound divisome components. We also identify at least two binding sites for FtsZ on EzrA and map regions of EzrA that are responsible for regulating FtsZ assembly. The individual repeats, and their linear organization, are homologous to the spectrin proteins that connect actin filaments to the membrane in eukaryotes, and we thus propose that EzrA is the founding member of the bacterial spectrin family.

\footnotetext{
${ }^{1}$ Institute for Cell and Molecular Biosciences, Newcastle University, Newcastle upon Tyne NE2 4HH, UK. ${ }^{2}$ Centre for Medical and Molecular Bioscience, University of Wollongong, Wollongong, New South Wales 2522, Australia. ${ }^{3}$ NUPPA, Devonshire Building, Newcastle University, Newcastle upon Tyne NE1 7RU, UK. ${ }^{4}$ The ithree Institute, University of Technology, Sydney, New South Wales 2007, Australia. † Present address: Helmholtz Centre for Infection Research, Helmholtz Institute for Pharmaceutical Research, Saarland University, 66123 Saarbrücken, Germany. Correspondence and requests for materials should be addressed to R.J.L. (email: r.lewis@ncl.ac.uk).
} 
B acterial cell division is directed by cytoskeletal proteins. The bacterial homologue of eukaryotic tubulin, Fts ${ }^{1}$, polymerises in the presence of $\mathrm{GTP}^{2}$ and is attached to the membrane at the mid-cell position to form the Z-ring ${ }^{3,4}$. The hydrolysis of GTP to GDP results in the dissociation of FtsZ polymers into monomers that disengage from the $Z$-ring ${ }^{5}$. Hence, the Z-ring is highly dynamic and constantly exchanges FtsZ molecules with the pool of its freely diffusible monomers ${ }^{6}$. The Z-ring is stabilized and anchored to the cytoplasmic membrane by interacting with FtsA, a bacterial homologue of actin, which is itself capable of polymerizing at the membrane ${ }^{7}$. FtsZ and FtsA recruit other cell division proteins, including cell wall synthesis enzymes and many proteins of uncharacterized function, to the mid-cell to form the mature divisome ${ }^{8}$. This large macromolecular assembly synthesizes the cell wall that will form the new poles of the cell after division ${ }^{9}$.

The polymerization of FtsZ into the Z-ring is a necessary and tightly regulated step in divisome assembly. The short C-terminal tail of FtsZ is essential for self-assembly and for interactions with its regulators ${ }^{3}$. Z-ring assembly is regulated by multiple, widely conserved cell division proteins, including FtsA, ZapA, the protease ClpX, SepF and EzrA. FtsA both recruits FtsZ filaments to the cell membrane and counteracts the clustering of FtsZ filaments on $i^{10}$. ZapA and SepF promote the bundling of FtsZ filaments into higher-order structures in vitro ${ }^{11-13}$. SepF additionally stabilizes the Z-ring at mid-cell in vivo ${ }^{14}$ by binding to both FtsZ and the membrane ${ }^{13}$.

By contrast, the integral membrane protein EzrA was identified as an inhibitor of FtsZ polymerization and its absence results in the inappropriate formation of multiple FtsZ rings, not only at mid-cell but also at the cell poles ${ }^{15,16}$. However, EzrA is also one of the first proteins to be recruited to the mid-cell by $\mathrm{FtsZ}^{8}$, suggesting it has an additional regulatory or scaffolding role in the formation of the divisome. As an inhibitor of FtsZ polymerization, EzrA is assumed to be required to maintain the dynamics of the Z-ring as it constricts during cell division ${ }^{16}$. Other evidence supports a positive role for EzrA in the assembly of the divisome. In Bacillus subtilis, EzrA recruits the major bi-functional, glycosyltransferase-transpeptidase peptidoglycan synthase, penicillin-binding protein 1 (PBP1, aka PonA) ${ }^{17}$ to the septum. Bacteria require the synthesis of new peptidoglycan to grow, and peptidoglycan must be deposited as cells divide to ensure a continuum of cell wall around both halves of the dividing cell. Consequently, PBPs that synthesize new cell wall material must localize to areas of cell growth or division. Deletion of $e z r A$ changes the cellular localization patterns of both FtsZ and PBP1, causing marked growth defects and alterations in cell morphology, division and elongation ${ }^{17,18}$. Generally, ezrA knockouts display a delay in cell division, resulting in cells about twice as long as wild type in B. subtilis ${ }^{13,15,19}$ and larger, spherical cells in Staphylococcus aureus ${ }^{18}$. The ezrA knockout has been described as lethal to $S$. aureus ${ }^{20}$, Streptococcus pneumoniae $^{21,22}$ and Listeria monocytogenes ${ }^{23}$, and thus constitutes a novel target for antibacterial agents. In B. subtilis, the concurrent knockout of ezrA with other divisome components including $z a p A^{11}$, sep $F^{14,17}$ or $g p s B^{17}$ causes severe growth defects. Therefore, EzrA plays key roles both as a regulator of cell division and in the maintenance of cell size and shape.

EzrA comprises a single, N-terminal trans-membrane (TM) spanning helix followed by an $\sim 60-\mathrm{kDa}$ cytoplasmic domain ${ }^{15}$, which sequence analysis suggests forms an $\alpha$-helical coiled-coil. To understand further the role of EzrA during cell division, we report the crystal structure of the intact, cytoplasmic domain of B. subtilis (Bs) EzrA at $3.95 \AA$ resolution. Its three-dimensional structure comprises five, $\sim 100$ amino-acid long repeats, each of which forms an anti-parallel triple helical bundle; these are arranged linearly to form a complete semi-circle with a diameter of $120 \AA$. The individual repeats and their linear head-to-tail arrangement are reminiscent of the spectrin family of eukaryotic cytoskeletal proteins that associate with actin filaments and membrane-bound proteins. Two copies of the spectrin repeats are also observed in a $2.6-\AA$ resolution structure of a $20-\mathrm{kDa}$ cytoplasmic fragment of EzrA from $S$. aureus $(S a)$, which has enabled direct molecular comparisons to be made between eukaryotic spectrin repeats and those found in EzrA. FtsZbinding sites on the BsEzrA arch are mapped and a binding site that inhibits FtsZ oligomerization is identified. We propose a role for EzrA that is analogous to that of spectrins in eukaryotes, in linking the dynamics of the bacterial cytoskeleton to the membrane-anchored components of the divisome, the proper localization of which is essential for the bacterial cell cycle.

\section{Results}

EzrA has a semi-circular structure. The crystal structure of B. subtilis EzrA lacking the $\mathrm{N}$-terminal 21 residue TM helix (that is, $B s$ EzrA $_{22-562}$ ) was solved by selenomethionine single wavelength anomalous dispersion (SAD) to a resolution of $3.95 \AA$. Despite extensive crystallization screening, the reliable diffraction limit could not be extended. However, through the use of highly redundant data, the experimentally phased electron density maps of $B s E_{22-562}$ (Supplementary Fig. 1a) enabled the building of

Table 1 | Crystallographic statistics.

\begin{tabular}{|c|c|c|}
\hline & BsEzrA 22-562 & SaEzrA $A_{24-214}$ \\
\hline \multicolumn{3}{|l|}{ Data collection } \\
\hline Space group & R32 & $P 4_{3} 2_{1} 2$ \\
\hline \multicolumn{3}{|l|}{ Cell dimensions } \\
\hline$a, b, c(\AA)$ & $222.1,222.1,183.5$ & $96.1,96.1,97.1$ \\
\hline$\alpha, \beta, \gamma\left({ }^{\circ}\right)$ & $90,90,120$ & $90,90,90$ \\
\hline \multirow{2}{*}{ Resolution $(\AA)$} & $29.2-3.95$ & $48.56-2.6$ \\
\hline & $(4.16-3.95)^{\star}$ & $(2.72-2.6)^{\star}$ \\
\hline$R_{\text {sym }}$ & $0.104(0.351)$ & $0.081(0.972)$ \\
\hline$R_{\text {pim }}$ & $0.03(0.266)$ & $0.031(0.368)$ \\
\hline$|/ \sigma|$ & $21.3(3.6)$ & $15.3(2.8)$ \\
\hline Completeness (\%) & $99.6(99.2)$ & $100(100)$ \\
\hline Redundancy & $22.4(4.0)$ & $15.3(2.8)$ \\
\hline $\begin{array}{l}\text { Anomalous } \\
\text { completeness (\%) }\end{array}$ & $99.2(95.7)$ & NA \\
\hline $\begin{array}{l}\text { Anomalous } \\
\text { redundancy (\%) }\end{array}$ & $11.4(2.5)$ & NA \\
\hline Solvent content (\%) & 82.2 & 74.7 \\
\hline \multicolumn{3}{|l|}{ Refinement } \\
\hline Resolution $(\AA)$ & $29.2-3.95$ & $48.56-2.6$ \\
\hline No. of reflections & 15,307 & 13,770 \\
\hline \multicolumn{2}{|l|}{ No. of atoms } & $0.209 / 0.249$ \\
\hline Protein & 4,264 & 1,586 \\
\hline Ligand/ion & 0 & 0 \\
\hline Water & 0 & 0 \\
\hline \multicolumn{3}{|l|}{$B$-factors } \\
\hline Protein $\left(\AA^{2}\right)$ & 155.6 & 88.4 \\
\hline \multicolumn{3}{|l|}{ RMSD } \\
\hline Bond lengths $(\AA)$ & 0.003 & 0.0102 \\
\hline Bond angles $\left(^{\circ}\right)$ & 0.785 & 1.34 \\
\hline $\begin{array}{l}\text { Molprobity geometry } \\
\text { score }\end{array}$ & $\begin{array}{l}2.6 \text { (98th } \\
\text { percentile) }\end{array}$ & 1.6 (100th percentile) \\
\hline Molprobity clash score & $\begin{array}{l}9.4 \text { ( } 97 \text { th } \\
\text { percentile) }\end{array}$ & $\begin{array}{l}1.15 \text { (100th } \\
\text { percentile) }\end{array}$ \\
\hline
\end{tabular}


$96 \%$ of the 540 amino acids in the structure. The only regions that could not be built were six residues at the $\mathrm{N}$-terminus and a loop between Ser177 and Asn190, which the sequence predicted to be disordered. Although only a few side chain features are visible at this resolution, the final refined model, which includes sidechains, has geometric and crystallographic refinement parameters that compare very favourably with other structures of similar resolution (Table 1). The final model correctly aligns methionine residues with the experimentally determined positions of the selenium atoms from the SAD analysis (Supplementary Fig. 1b).

Overall, the $B s \mathrm{EzrA}_{22-562}$ monomer is a curved rod, forming a complete semicircle with a diameter of $120 \AA$. Groups of three $\alpha$-helices pack together along its length (Fig. 1a), except at the C-terminus where there is a four-helix bundle. The formation of a semicircle confers the potential of the C-terminal four-helix bundle to interact with the membrane at the same time that the $\mathrm{N}$-terminus of EzrA is embedded in it through its N-terminal TM helix; this can be modelled trivially by manually extending the $\mathrm{N}$-terminal $\alpha$-helix of repeat 1 (Supplementary Fig. 1c). An antiparallel dimer is observed in the $B s E_{2 r A} A_{22-562}$ crystal lattice as a result of crystallographic symmetry (Supplementary Fig. 2a). The dimerization of $B s E_{22} A_{22-562}$ in the crystal buries some 3,300 $\AA^{2}$ of surface area ( $\sim 9 \%$ of the total) in an extensive, molecule-long interface. The anti-parallel dimer could not be detected in solution by sedimentation velocity, which indicates that the monomer and an oligomer of unknown arrangement are in equilibrium (Supplementary Fig. 2b). However, bacterial twohybrid analysis in vivo shows an interaction between the $\mathrm{N}$ - and $\mathrm{C}$-terminal regions of BsEzrA (Supplementary Fig. 2c), which is consistent with the anti-parallel dimer seen in the crystals and which is the only oligomeric arrangement in the crystal that would enable the N-terminal TM helices of both subunits to insert into the same face of a membrane in vivo.

To obtain higher resolution structural information on EzrA, several fragments from different EzrA homologues were subjected to crystallization. A 190 residue $\mathrm{N}$-terminal fragment of S. aureus EzrA, SaEzrA $24-214$ (that also lacks its N-terminal TM helix), yielded crystals that diffracted to $2.6 \AA$ resolution. Its structure (Fig. 1b, Supplementary Fig. 1d) was solved by molecular replacement using model phases from the equivalent portion of $B s \mathrm{EzrA}_{22-562}$, and was refined to a final crystallographic $R_{\text {free }}$ of 0.25 (Table 1). The structures of $S a E_{2 r A} A_{24-214}$ and $B s E_{2 r A} 22-562$, which share only $20 \%$ sequence identity, superpose with root mean square deviations (RMSDs) of $2.4 \AA$ on 154 paired $C \alpha$ atoms (Supplementary Fig. 3a). The $B s E_{2 r A} A_{22-562}$ and the shorter $S a$ EzrA $_{24-214}$ rods comprise multiple copies of the same $\sim 100$ amino acid, triple helical bundle; $B \mathrm{sEzrA}_{22-562}$ contains five such repeats and $\mathrm{SaEzrA} 24-214$ contains just two. The individual repeating units can all be superimposed with RMSDs of between 1.5 and $2.3 \AA$ on paired C $\alpha$ atoms (Supplementary Fig. $3 b$ ).

Structural homologues of EzrA occur only in eukaryotes. A search for structural homologues of $B s$ ErA $_{22-562}$ using PDBefold $^{24}$, DALI $^{25}$ and BLAST-3D ${ }^{26}$ using default matching criteria did not identify any structures that superimposed convincingly along the entire length of the structure; only short segments could be aligned onto structural homologues. Representative matches from a BLAST-3D search included the eukaryotic cytoskeletal proteins vinculin ${ }^{27}$ and $\alpha$-actinin ${ }^{28}$, the nuclear importers karyopherin ${ }^{29}$ and importin ${ }^{30}$ and four-helical coiled coils such as the bacterial chemotaxis receptor, $\mathrm{CheA}^{31}$ (Supplementary Fig. 4a). The same searches were repeated with the structure of $S a \operatorname{ErA}_{24-214}$ as the query. PDBefold identified five matches, all of which resembled the hits from the BLAST-3D search with $B s E_{2 r A} \mathrm{~A}_{22-562}$; one match was to vinculin and the a

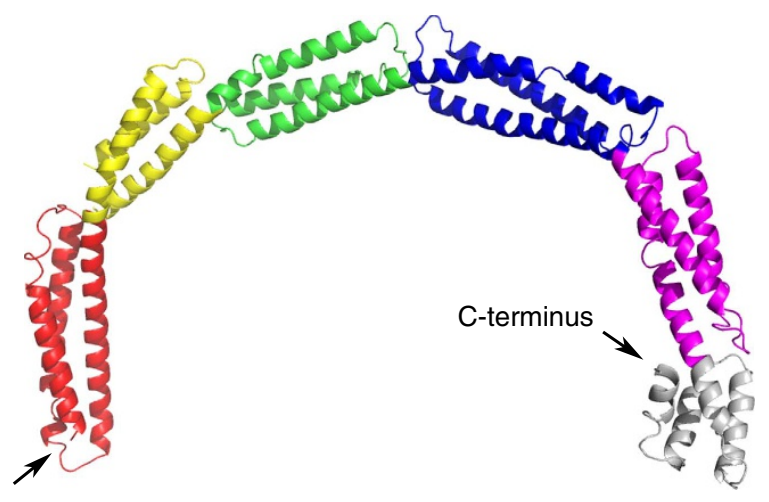

$\mathrm{N}$-terminus

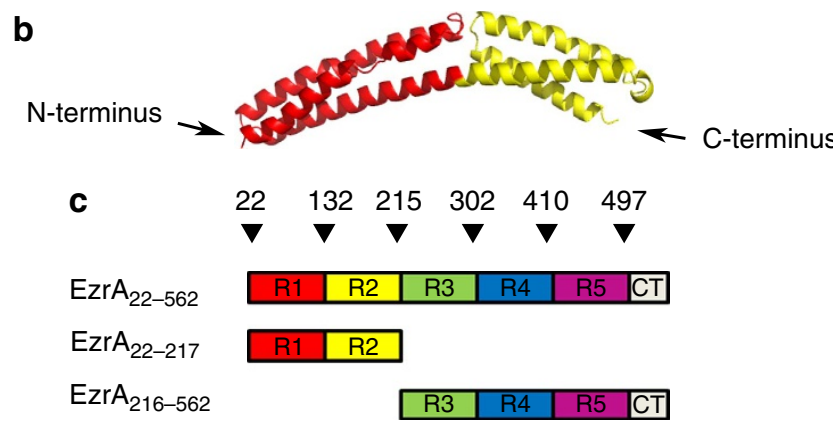

Figure 1 | Structure of the cytoplasmic domain of EzrA. (a) Ribbon diagram of the BsEzrA $A_{22-562}$ monomer coloured by triple helical bundle repeats from $\mathrm{N}$ - to $\mathrm{C}$-terminus, red, yellow, green, blue and magenta. The C-terminal, four-helix bundle is coloured silver. This and all other structure representations were made in PyMOL (http://www.pymol.org). (b) Ribbon diagram of the SaEzrA $24-214$ monomer, coloured as in a. (c) Schematic illustration of the BsEzrA fragments used in this study, with blocks (numbered R1-R5) representing the spectrin repeat units and the C-terminal four-helix bundle (labelled CT) and coloured as in a. The N- and $\mathrm{C}$-termini and the junctions between spectrin repeats are numbered above the schematic.

other four were to members of the spectrin superfamily to which $\alpha$-actinin belongs.

Although EzrA structures could be aligned partially onto other proteins that also have an up-down-up arrangement of helices, the match with the spectrin superfamily of structures stood out as most significant. Despite having an up-down-up helix configuration, vinculin, karyopherin, importin and CheA all lack discrete triple-helical bundles; in all of these protein families, each helix packs intimately against three, rather than two, other helices. In addition, although DALI searches matched both EzrA structures with other rod-shaped triple helical bundles, such as BAR domain $^{32}$ (Supplementary Fig. 4a), in these proteins there is only a single, triple helical bundle that is longer ( $>150$ amino acids) than the repeat unit in EzrA. The linear head-to-tail arrangement of interconnected $\sim 100$ amino-acid triple helical bundles appears to be unique to spectrins and to EzrA. Finally, no matter which search tool was used, the C-terminal four-helix bundle of EzrA superimposed only weakly on matching structures in the PDB, but it is notable that it has a superficial resemblance to the helical bundle fold of the actin-binding calponin homology domains ${ }^{33}$, which can be found at the termini of spectrin-type proteins.

EzrA is the founding member of the bacterial spectrin family. To our knowledge, the structural similarity of EzrA or any other bacterial protein to spectrins had not been predicted. From the logic below, we conclude that EzrA is the founding member of a 
new family of bacterial spectrin-like proteins: (i) the eukaryotic structural homologues of EzrA share the same extended rod-like character, comprising repeating units of $\sim 100$ amino acids arranged as triple helical anti-parallel bundles in a head-to-tail linear array (Fig. 2a, Supplementary Fig. 4b). Eukaryotic spectrins contain 4-20 of these units, called spectrin repeats, whereas EzrA contains 5 of them. (ii) Four of the five $B s E_{2 r A}{ }_{22-562}$ spectrin repeats and the two in $S a$ EzrA $_{24-214}$ can be superimposed on representative eukaryotic spectrin repeats with RMSDs of between 1.9 and $2.6 \AA$ on matched $\mathrm{C} \alpha$ atoms (Fig. 2b); this is a high degree of similarity that is comparable to RMSDs among the EzrA spectrin repeats. Moreover, each superimposition accounts for greater than $70 \%$ of the EzrA spectrin repeat with paired amino acids coming from all three of the helices. (iii) The structural similarity of EzrA to the spectrin superfamily extends to the junctions and interfaces among adjacent repeats. As with all other crystallized spectrins, the repeat junctions in EzrA are formed from long continuous $\alpha$-helices that form the third helix of one repeat and the first helix of the next. As also observed in several spectrins ${ }^{34-36}$, the $\mathrm{SaEzrA} \mathrm{E}_{24-214}$ structure reveals intimate interactions at the interface between repeats involving the linker helix and loop residues. For example, the linker residues Tyr135 and Ile131 interact with loop residues Thr62, Leu56 and Tyr193 (Supplementary Fig. 5a,b). (iv) Although there are multiple examples of spectrin structures containing dual repeats, arranged like EzrA in a head-to-tail linear arrangement (Figs 1a and 2a, Supplementary Fig. 4b), the semi-circular curvature (Figs 1a and 2a) of $B s$ EzrA $_{22-562}$ is unique. The principal axes of adjacent repeats in $B s E_{22-562}$ intersect with angles of $37,38,33$ and $53^{\circ}$, resulting in the pronounced overall bend. The angle between the two repeats in $\mathrm{SaEzrA} \mathrm{En}_{24-214}$ is $34^{\circ}$, suggesting that full-length EzrA from $S$. aureus will also have a pronounced bend. The magnitude of these angles in EzrA is similar to that between erythrocytic $\beta$-spectrin repeats 14 and 15 (ref. 35), which is $37^{\circ}$ (Fig. 2c). Furthermore, a study of the six-repeat plakin domain of desmoplakin in solution by small-angle X-ray scattering was consistent with the plakin adopting an overall L-shape ${ }^{39}$, but $B s$ EzrA $_{22-562}$ is the first structure with a sufficient bend between enough repeats to generate a full semi-circle. The absence of longer chains of spectrin repeats from the PDB may simply reflect a crystallization bias towards the shorter, relatively rigid, dual spectrin repeats that predominate in the database.

Nevertheless, there are facets of the EzrA structure that differentiate it from spectrins. For instance, there is no sequence homology between EzrA and any spectrin, although short segments of both proteins share a heptad repeat periodicity of hydrophobic amino acids (Supplementary Fig. 6). Although the orientations of the three $\alpha$-helices in the EzrA repeats are similar to those of spectrin repeats (Fig. $2 \mathrm{a}, \mathrm{b}$ ), the relative positions and therefore the connectivities of the helices are different (Fig. 2d).

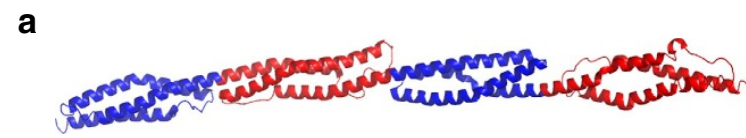

b

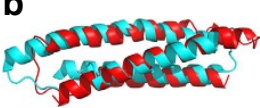

BsEzrA $24-129$

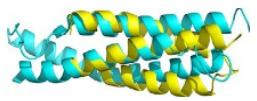

$B s \mathrm{EzrA}_{130-217}$

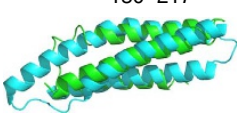

BsEzrA $220-303$

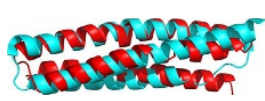

SaEzrA $24-133$

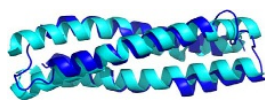

BsEzrA $304-409$

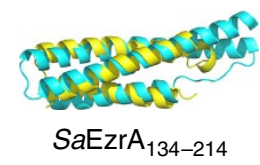

SaEzrA $134-214$
C

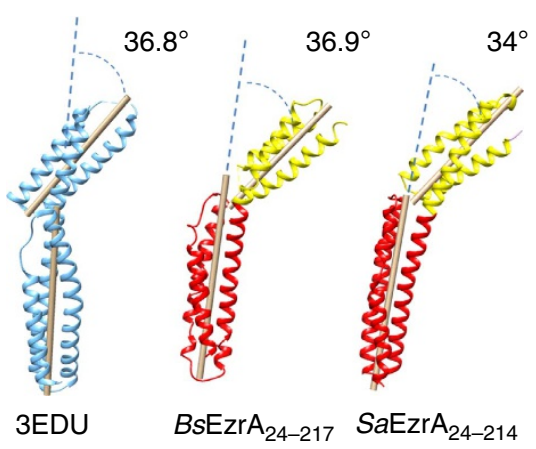

d

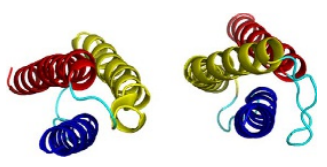

BsEzrA $24-129 \quad 1 \mathrm{HCl}_{630-746}$

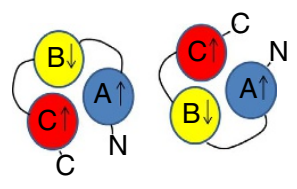

EzrA Spectrin

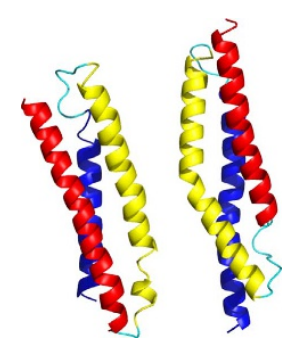

$B s$ ErA $_{24-129} 1 \mathrm{HCl}_{630-746}$

Figure 2 | Comparison of EzrA with spectrin family proteins. (a) Structure of the spectrin protein $\alpha$-actinin (PDB 1HCI), with alternating triple helical

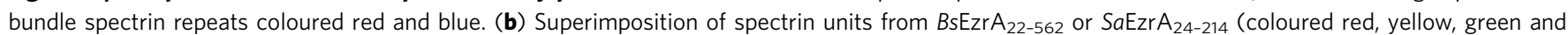
blue, as in Fig. 1a) onto representative spectrin units coloured cyan from erythroid spectrin (top; PDB 3KBT), $\alpha$-actinin (middle; PDB $1 \mathrm{HCI}$ ) and brain spectrin (bottom; PDB 1U5P). (c) The angle between spectrin repeats 1 and 2 in $B_{s E z r A} A_{22-562}$ is $37^{\circ}$ and in SaEzrA $24-214$ it is $34^{\circ}$; the angle is also $37^{\circ}$ between erythrocytic $\beta$-spectrin repeats 14 and 15 (PDB 3EDU). (d) Top-Orthogonal views of the relative positioning and different connectivity of the three $\alpha$-helices in EzrA spectrin repeat 1 (residues 24-129) and in the fourth repeat (residues 630-745) of the rod domain of $\alpha$-actinin (PDB 1HCI). The $\mathrm{N}$-terminal helix in the bundle (a) is coloured blue, the middle helix (b) yellow and the C-terminal helix (c) red. Bottom-Schematic illustration of the different connectivity in the EzrA and spectrin three-helix bundles, viewed along the helix axes. An up arrow (helix a, c) represents an 'up' helix viewed along the helix axis in an $\mathrm{N}-\mathrm{C}$ direction ( $\mathrm{N}$-terminus of helix axis above plane of page). A down arrow represents a 'down' helix viewed in the opposite direction. 
For example, the first 'up' helix in the EzrA repeat, helix A (blue, Fig. 2d), is superimposed on the third 'up' helix in the spectrin repeat from $\alpha$-actinin, helix C (red, Fig. $2 \mathrm{~d}$ ). The second 'down' helix in the EzrA repeat is superimposed well on the $\alpha$-actinin equivalent (helix B, coloured yellow in Fig. $2 d$ ), whereas the third 'up' helix in the EzrA repeat, helix $C$, is superimposed on the first 'up' helix in the $\alpha$-actinin spectrin repeat, helix A. In other words, the left-handedness of the connectivity between helices in eukaryotic spectrin repeats (helices $\mathrm{A}-\mathrm{C}$ are arranged in a counter-clockwise manner when viewed end-on) is reversed to be right-handed in EzrA (helices A-C are arranged clockwise).

Direct interaction of EzrA with FtsZ. To map which regions of BsEzrA interact with $B$. subtilis FtsZ (BsFtsZ), a qualitative crosslinking approach using 1-ethyl-3-(3-dimethylaminopropyl) carbodiimide (EDC), which conjugates amines directly to carboxyl groups, was combined with quantitative surface plasmon resonance (SPR) measurements. EDC-mediated zero-length crosslinks were found between BsFtsZ and the BsEzrA $22-562$ fragment, but not to unrelated control proteins (Fig. 3a). To determine more precisely the crosslinking site within EzrA, the $B s E_{22} A_{22-562}-B s$ FtsZ adduct was cleaved site-specifically into defined fragments using the cysteine-specific cleavage reagent 2-nitro-5-thiocyanatobenzoic acid (NTCB). For this, a $B s E_{22-562}$ variant (K135C/C222A) with a single cysteine between spectrin repeats 1 and 2 was prepared (Fig. 3b). Cleavage of the $B s \operatorname{EzrA}_{22-562}(\mathrm{~K} 135 \mathrm{C} / \mathrm{C} 222 \mathrm{~A})-B s \mathrm{FtsZ}$ adduct with NTCB yielded a fragment consistent with the attachment of $B s F t s Z$ to the N-terminal region of BsEzrA (residues 22-134) comprising the first spectrin repeat (Fig. 3c).

Guided by limited proteolysis studies and the crystal structure, two truncated BsEzrA fragments were produced. BsEzrA $22-217$ encompassed repeats one and two and $B s \mathrm{EzrA}_{216-562}$ included the other three and the C-terminal domain (Fig. 1c). BsEzrA $22-217$ bound to an SPR chip surface coated with immobilized BsFtsZ with fast kinetics, enabling equilibrium binding levels to be measured (Fig. 4a). Fitting the binding isotherm to a 1:1 binding model yielded a dissociation constant $\left(K_{\mathrm{d}}\right)$ of $\sim 13 \mu \mathrm{M}$ for the $B s$ FtsZ:BsEzrA $22-217$ complex (Fig. 4b). The BsEzrA $216-562$ fragment also bound to the BsFtsZ-coated chip, but with considerably slower kinetics (Supplementary Fig. 7a), and fitting the sensorgrams to a $1: 1$ binding model yielded a $K_{\mathrm{d}}$ of $2 \mu \mathrm{M}$. The specificity of the observed interactions is supported by the lack of any interaction of either $B s \mathrm{EzrA}_{131-217}$ or bovine serum albumin with the FtsZ chip surface under equivalent buffer conditions (dashed cyan sensorgrams, Fig. 4a and Supplementary Fig. 7c).

The binding of both $B s \mathrm{EzrA}_{22-217}$ and BsEzrA $216-562$ to BsFtsZ implies that there are either two distinct FtsZ-binding sites on an EzrA monomer or a single site that extends across the interface between repeats 2 and 3 . However, to be consistent with the crosslinking of $B s$ Fts $Z$ to repeat 1 , a single binding site at the interface between repeats 2 and 3 would need to extend also to spectrin repeat 1 , implying an intimate association of FtsZ with repeat 2. However, a fragment encompassing only repeat 2, BsEzrA $131-217$, showed no detectable interaction with immobilized BsFtsZ (red dashed traces, Fig. 4a) in SPR, despite it having appreciable secondary and tertiary structure content (Supplementary Fig. 8a,b). Therefore, it seems most likely that there are at least two distinct FtsZ interaction sites on an EzrA monomer, one of which is in spectrin repeat 1 .

Effect of EzrA on FtsZ oligomerization. The BsEzrA-BsFtsZ interaction was analysed to map regions of EzrA responsible for the inhibition of FtsZ oligomerization. GTP-induced increase in

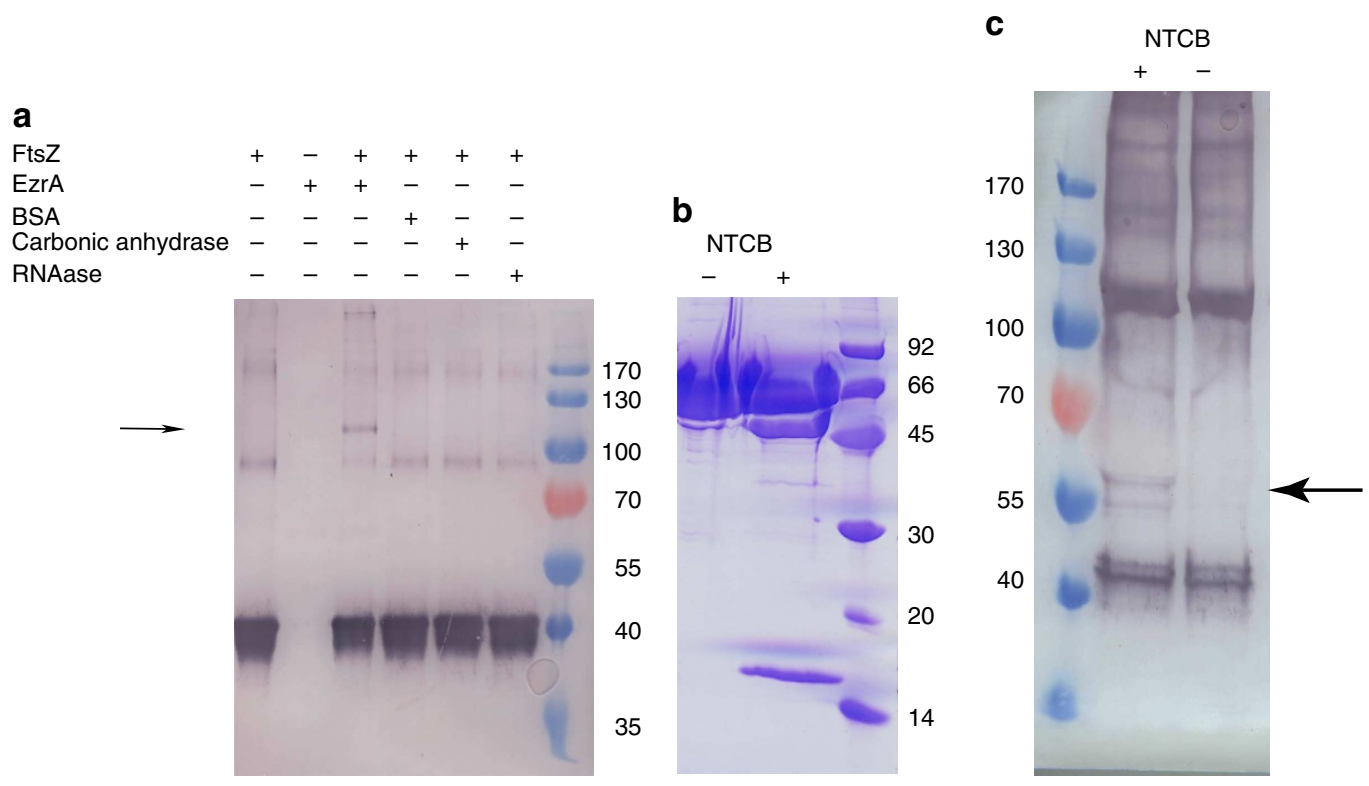

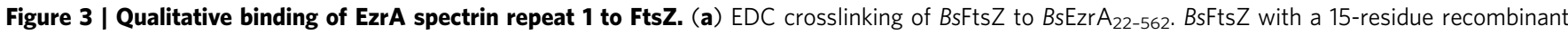
$\mathrm{N}$-terminal $\mathrm{T7}$ epitope tag was incubated with $B_{s} E_{2 r} \mathrm{~A}_{22-562}$ or various control proteins with the crosslinking agent EDC. The crosslinking reactions were analysed by SDS-PAGE and by western blotting to detect the T7 epitope tag. Only the reaction containing BsEzr $\mathrm{A}_{22-562}$ and $B s F t s Z$ yields a unique product (highlighted with an arrow) with a mobility consistent with a 1:1 EzrA $22-562$ :FtsZ adduct of mass $\sim 100 \mathrm{kDa}$. The units of the masses listed at the side of this and the other two panels in this figure are in $\mathrm{kDa}$. (b) Cysteine-specific fragmentation of the BsEzrA-BsFtsZ adduct between spectrin repeats 1 and 2. The BsEzrA $22-562$ mutant $\mathrm{K} 135 \mathrm{C} / \mathrm{C} 222 \mathrm{~A}$ has only one cysteine, at the interface between spectrin repeats 1 and 2. NTCB cleavage of isolated, purified $\mathrm{K} 135 \mathrm{C} / \mathrm{C} 222 \mathrm{~A}$ generates fragments of the expected size (16 kDa for residues 22-134 plus the $\mathrm{N}$-terminal hexahistidine expression tag; $49 \mathrm{kDa}$ for residues 135-562) on SDS-PAGE. (c) Cleavage of the BsEzrA $22-562 \mathrm{~K} 135 \mathrm{C} / \mathrm{C} 22 \mathrm{~A}-$ BsFtsZ crosslinked adduct with NTCB. The cleavage product was analysed by SDS-PAGE and by western blotting to detect the T7 epitope tag on FtsZ. After NTCB cleavage, a band (marked with an arrow) appears close to the 55-kDa marker, which is consistent with the 16-kDa N-terminal BsEzrA fragment (residues 22-134) crosslinked to the 40-kDa BsFtsZ. 

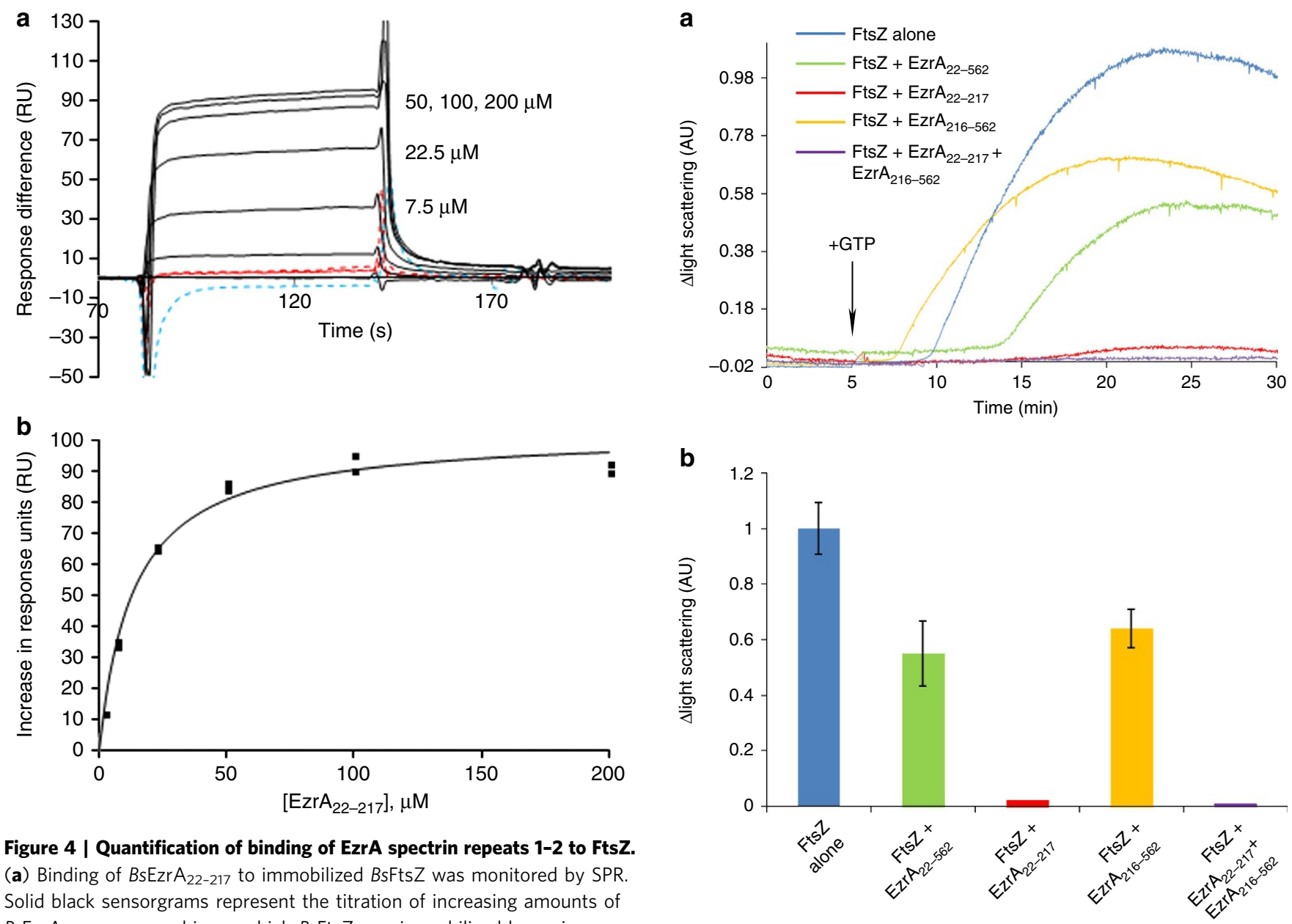

Figure 4 | Quantification of binding of EzrA spectrin repeats 1-2 to FtsZ (a) Binding of BsEzrA $A_{22-217}$ to immobilized BsFtsZ was monitored by SPR. Solid black sensorgrams represent the titration of increasing amounts of

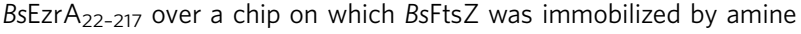
coupling. The dashed cyan sensorgram represents an injection of $50 \mu \mathrm{M}$ bovine serum albumin (BSA) over the same surface under the same conditions; the dashed red sensorgrams represent injections of 25, 50 and $100 \mu \mathrm{M}$ of the $B s E_{2 r} A_{131-217}$ fragment. (b) The response units of BsEzrA $22-217$ binding the BsFtsZ surface as a function of injected protein were fit with a curve corresponding to a 1:1 binding interaction, yielding a $K_{d}$ of $13.7 \pm 1.5 \mu \mathrm{M}$. The actual binding process may be more complex than a simple 1:1 interaction because of the self-association of the $B s E_{2 r} A_{22-217}$ fragment, apparent from size exclusion chromatography-multi angle laser light scattering analysis (SEC-MALLS) (see Supplementary Fig. 7b).

light scattering provides a measure of the polymerization of FtsZ monomers into filaments and the bundling of filaments into higher order structures; we refer to these processes collectively as 'oligomerization'. Previous studies have shown that a twofold excess of BsEzrA over BsFtsZ reduced FtsZ oligomerization between two- and tenfold ${ }^{16,38}$. The $\mathrm{pH}$ was increased from 6.5, which was used in previous studies with this assay, to 7.3 , to reduce the aggregation of $B s \mathrm{EzrA}_{22-562}$. Under these conditions, a twofold excess of BsEzrA $\mathrm{A}_{22-562}$ inhibited BsFtsZ oligomerization about twofold (Fig. 5a,b), consistent with previous studies ${ }^{16,38}$. Remarkably, identical concentrations of $B s \mathrm{EzrA}_{22-217}$ in this assay inhibited $B s$ FtsZ oligomerization almost completely (Fig. $5 \mathrm{a}, \mathrm{b}$ ), whereas a twofold excess of $B s \mathrm{EzrA}_{216-562}$ reduced $B s \mathrm{FtsZ}$ oligomerization by about one-third. An equimolar mixture of $B s E z r A_{22-217}$ and $B s E_{2 r A} A_{216-562}$ also completely inhibited $B s$ FtsZ assembly (Fig. 5a,b). The stronger inhibition of BsFtsZ assembly by the smaller $B s \mathrm{EzrA}_{22-217}$ fragment in comparison to $B s E_{2 r A} A_{22-562}$ may reflect the masking of FtsZ-interacting sites by the oligomerization of $B s E_{22} A_{22-562}$; size exclusion

Figure 5 | Inhibition of FtsZ oligomerization by EzrA fragments.

(a) The GTP-induced oligomerization of FtsZ was monitored by light scattering. All samples contained $12.5 \mu \mathrm{M} \mathrm{BsFtsZ}$ and $25 \mu \mathrm{M}$ of the indicated BsEzrA fragment. The arrow corresponds to the time when GTP was added to a final concentration of $1 \mathrm{mM}$. (b) The average GTP-induced increase in light scattering in the presence of the BsEzrA fragments. The results shown are the mean \pm s.d. from three independent measurements. The $B s E z r A_{22-217}$ fragment, encompassing spectrin repeats 1-2, reduces FtsZ polymerization to undetectable levels.

chromatography of $B s \mathrm{EzrA}_{22-562}$ revealed that it formed an oligomer in the low salt buffer conditions used in the light scattering assay (Supplementary Fig. 8c). The relative levels of inhibition of BsFtsZ assembly by different BsEzrA fragments must be interpreted cautiously, but the marked inhibition by $B s$ EzrA $_{22-217}$ points once more to a functionally important interaction of the N-terminal region of EzrA with FtsZ.

To ascertain whether the marked inhibition of BsFtsZ oligomerization by $B s \mathrm{EzrA}_{22-217}$ reflects direct inhibition of FtsZ polymerization into filaments, or the blocking of filament bundling into higher order structures, its effect on the GTPase activity of $B s F t s Z$ was examined. Head-to-tail polymerization of FtsZ into filaments is dependent upon GTP binding ${ }^{39}$, and dissociation of FtsZ polymers into monomers is dependent upon GTP hydrolysis ${ }^{5}$. Therefore, either the inhibition of GTP binding or the stimulation of the GTPase activity could result in the inhibition of FtsZ head-to-tail polymerization by EzrA. The presence of $B s E z r A_{22-217}$ did not markedly affect the binding of the fluorescent BODIPY-GTP- $\gamma$-S to BsFtsZ (Supplementary Fig. 9a), a result that is consistent with the very slight reduction 
(1.6-fold) in GTP affinity for BsFtsZ in the presence of BsEzrA measured previously ${ }^{40}$. The GTPase activity of $B s F t s Z$ was measured in the presence and absence of $B s E_{22-217}$, to determine whether EzrA affected GTP hydrolysis and thus FtsZ oligomerization. Both $B s$ EzrA $_{22-562}$ and $B s$ EzrA $_{22-217}$ had marginal effects on GTP turnover in a coupled GTPase assay (Supplementary Fig. 9b) and in a malachite green assay that monitored release of free phosphate (Supplementary Fig. 9c). It would thus seem that EzrA has little impact on the binding of GTP by FtsZ or on its hydrolysis.

Finally, we examined the impact of $B s \mathrm{EzrA}_{22-217}$ on the oligomerization of $B s \mathrm{FtsZ}_{1-366}$, which lacks the C-terminal 17 amino acids required for bundling of FtsZ filaments ${ }^{41,42}$. Consistent with previous observations ${ }^{41}$, the change in light scattering on adding GTP to $B s \mathrm{FtsZ}_{1-366}$ was reduced markedly, by $\sim 15$-fold relative to wild-type BsFtsZ (Supplementary Fig. 10a). As has been observed previously with $B s$ EzrA $_{22-562}$ (ref. 43), EzrA $22-217$ had no impact on the residual GTP-induced oligomerization activity of $B s F t s Z_{1-366}$ (Supplementary Fig. 10). These results suggest that EzrA does not affect the head-to-tail polymerization of FtsZ into filaments but instead largely acts by preventing the bundling of FtsZ filaments into higher-order assemblies.

\section{Discussion}

Spectrins are ubiquitous proteins involved in the organization of the cytoskeleton and membrane processes in animals. A typical spectrin has an elongated molecular shape due to multiple, connected repeats of three anti-parallel $\alpha$-helices, which are slightly curved and coil around each other. Individual spectrin repeats are approximately $50 \AA$ long and $20 \AA$ wide and are similar in length (99-122 amino acids). Their primary aminoacid sequences differ significantly, but the bundling of the helices is stabilized by regularly spaced hydrophobic side chains. EzrA shares all of these features with the spectrin superfamily of proteins, and the structural similarity of the EzrA repeats with those of other spectrin-like proteins is striking (Fig. 2). Although many proteins are known to comprise bundles of antiparallel $\alpha$-helices, EzrA is, to the best of our knowledge, the only bacterial protein that is formed from serial spectrin-like repeats.

Structure-based sequence alignments do not show any meaningful sequence homology either among the five EzrA repeats, or between EzrA and spectrins. Such a lack of sequence conservation is not without precedence within the spectrin superfamily ${ }^{44}$. The spectrin repeat is a degenerate sequence established predominantly on the heptad repeat pattern of hydrophobic amino acids in the primary sequence, a characteristic of lefthanded helical supercoiled proteins ${ }^{45}$. The degeneracy is exemplified by the observation that there are only four invariant residues in an alignment of the four spectrin repeats in human $\alpha$-actinin 2, one invariant residue in an alignment of the ten spectrin repeats in the human Duchenne muscular dystrophy protein and none in the seventeen spectrin repeats of human erythrocyte $\beta$-spectrin. Therefore, even the tryptophan in the first helix in the repeat, originally described as critical for thermodynamic stability of the spectrin repeat ${ }^{46,47}$, is not strictly maintained. The first and third repeats in EzrA each contain a highly conserved proline residue in helix A; Pro45 of SaEzrA in repeat 1 (Supplementary Fig. 11a) is conserved in eight out of nine phylogenetically diverse paralogues, whereas Pro228 (Pro227 in BsEzrA) in repeat 3 (Supplementary Fig. 11b) is conserved in all of them. Although sequence analyses of spectrins have identified that a proline is found frequently in helix $\mathrm{B}^{48}$, neither helix $\mathrm{A}$ nor $\mathrm{C}$ has been reported to be interrupted by proline residues.
There is significant variation in the relative orientation of adjacent repeat units in spectrin structures ${ }^{35,49}$. However, EzrA is the first spectrin-like repeat-containing structure in which there is a pronounced enough bend between a sufficient number of repeats to form a complete semicircle. Molecular dynamics studies suggest the flexibility of the junction regions in spectrins is constrained appreciably, with the force constants for changing the angle between adjacent repeats determined ${ }^{36}$ to be between 20 and $60 \mathrm{kcal} \mathrm{mol}^{-1} \mathrm{rad}^{-2}$. A considerable input of energy is thus likely to be necessary to convert the semicircular EzrA into a straight rod. The solvent contents of the EzrA crystals are unusually high (BsEzrA $22-562$ 80\%; $\mathrm{SaEzrA}_{24-214} 74 \%$ ); $>80 \%$ of all entries in the PDB have a solvent content $<60 \%$ and only $1.1 \%$ have a solvent content $>75 \%$. The high solvent content of these crystals does not concur with the release of sufficient energy during crystallization to drive thermodynamically unfavourable events. Therefore, the semicircular shape is highly unlikely to represent an artefact induced by crystal packing. The extended curved structure is a property EzrA shares with SepF, an FtsZ bundling protein required for normal septum formation. $B$. subtilis SepF assembles into polymeric $\operatorname{arcs}^{13}$ and rings ${ }^{12}$ with a diameter $(55 \mathrm{~nm})$ larger than that of the EzrA semicircle $(12 \mathrm{~nm})$. FtsZ filaments are proposed to bind on the outside of the SepF curve, whereas the inner face interacts with the surface of the invaginating septum membrane ${ }^{13}$. An analogous intimate association of the much smaller inner face of the EzrA semicircle with the membrane in vivo would require extreme curvature of the lipid bilayer, which has not been reported to date.

A question raised by the $B s E_{22-562}$ structure is the orientation of full-length EzrA relative to the lipid bilayer. A tilted orientation of the EzrA arch with respect to the membrane could result from the curvature of the membrane, the bending of the TM helix or flexibility in the junction between the TM helix and the cytoplasmic domain. The conserved proline in helix 1 of repeat 1 (Pro45 in S. aureus EzrA) may confer some additional flexibility in the junction between the body of the cytoplasmic domain and the membrane. However, an intimate association of the EzrA cytoplasmic domain with the surface of the membrane, as seen in the curve-shaped helical BAR domain proteins ${ }^{32}$, is not supported by electrostatic potential calculations on either of the EzrA structures presented here. A tilted orientation of the arch would position the C-terminal four-helix bundle away from the surface of the membrane. This domain contains a conserved motif between residues 505 and 511, termed the 'QNR patch' (Supplementary Fig. 1c), that is important for mid-cell localization ${ }^{38}$. The QNR patch has been proposed recently to bind directly to FtsZ, based upon the inhibition of FtsZ oligomerization by different EzrA fragments in a light scattering assay in vitro ${ }^{50}$

In the simplest model for the orientation of EzrA, the TM helix would form a straight extension of the first few turns of helix A of the first spectrin repeat; secondary structure predictions suggest a continuous uninterrupted helix extends into the first helix in the cytoplasmic domain. This orientation would result in the cytoplasmic domain of EzrA forming an arch with the C-terminal helical bundle positioned close to the membrane surface (Supplementary Fig. 1c). Although the sequence register in this region of the structure must be regarded cautiously, because of the paucity of methionine residues in this region of the sequence, this model would position key solvent-exposed residues in the QNR patch (R510, R512 and F511) to interact with the membrane, and preclude their direct interaction with Z-ring FtsZ polymers (Supplementary Fig. 1c). This suggests rather that the QNR patch controls EzrA localization through direct interaction with the membrane or specific membrane-associated protein(s). 
An arch over the membrane could allow EzrA to regulate FtsZ oligomerization by trapping FtsZ filaments in the gap between the membrane and the inside of the EzrA curve. Molecular modelling with the Methanococcus janaschii FtsZ dimer ${ }^{51}$ and the Thermotoga maritima FtsA:FtsZ complex ${ }^{7}$ reveals there is sufficient space beneath the arch, in both monomeric and dimeric forms of EzrA, to accommodate an FtsZ filament or even to simultaneously accommodate FtsA-mediated membraneanchored FtsZ and FtsA protofilaments (Fig. 6). Trapping FtsZ protofilaments inside the arch is intuitively an effective means to disrupt lateral interactions between protofilaments and could additionally anchor contractile protofilaments to the membrane. An individual filament inside the arch cannot engage in lateral interactions with filaments outside of it (Fig. 6), providing an additional level of Z-ring regulation. The ability of the arch to accommodate FtsZ protofilaments could be modulated depending on the cellular location of EzrA by the curvature of the cell membrane and/or by competing interactions of EzrA with other divisome components such as GpsB. In addition, or alternatively, FtsA and FtsZ may interact with the outside face of the EzrA arch only, leaving the inner surface for mediating alternative protein-protein interactions.

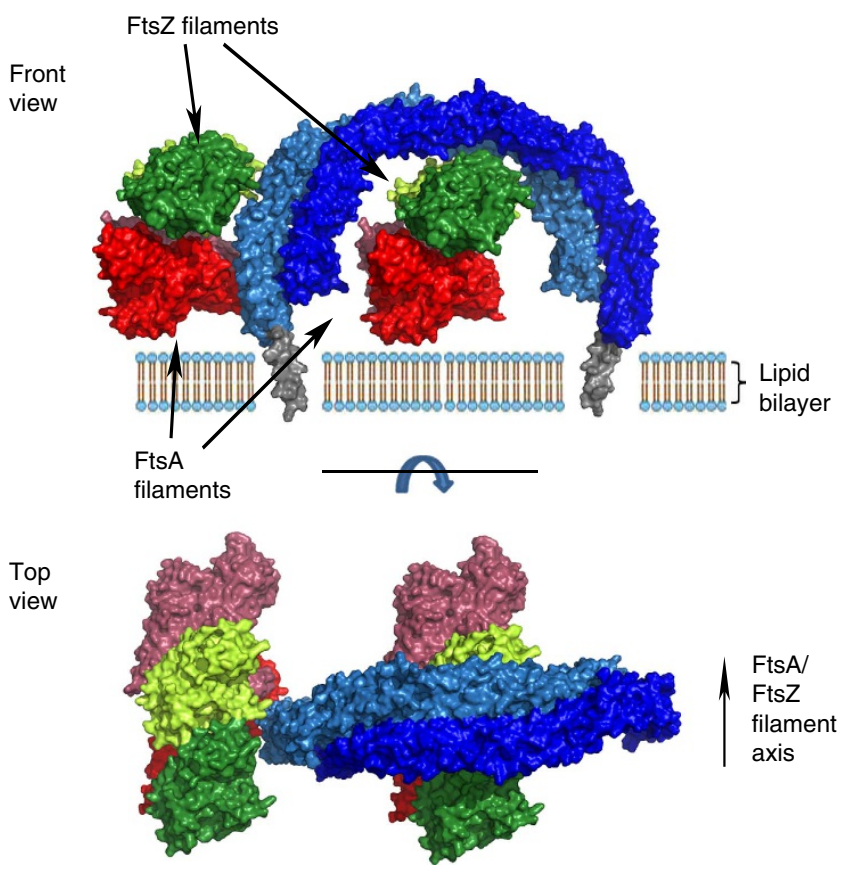

Figure 6 | Model of the interaction of EzrA with the cytoskeleton at the membrane surface. Model of FtsZ and FtsA protofilaments situated both alongside and beneath the inner face of the arch-shaped EzrA molecule (here represented as the crystallographic anti-parallel dimer, component subunits coloured light and dark blue). The modelled TM helix of EzrA is coloured grey. The coordinates of T. maritima FtsA bound to a C-terminal fragment of FtsZ (PDB 4A2A) and the M. jannaschii FtsZ dimer (PDB 1W59) and $B$. subtilis Fts $Z$ monomer (PDB 2VAM) were used to model FtsA (light and dark red) and FtsZ (light and dark green) protofilaments, respectively. FtsA has been oriented to place the membrane-anchored C-terminus close to the lipid bilayer and its binding site for FtsZ near the FtsZ filament. The FtsZ filament has been positioned to place its C-terminal FtsA-binding region (disordered in FtsZ crystal structures) close to the FtsA filament. An FtsZ filament trapped beneath the EzrA arch cannot bundle together with filaments outside the arch. Although the crystallographic dimer is represented, the same effect on bundling could be made by EzrA monomers.
A bacterial origin for the eukaryotic cytoskeleton has been suggested previously, in part, because of the similarity in structure and function of FtsZ to tubulin and FtsA to actin, although the primary amino-acid sequences are poorly related ${ }^{1,52,53}$ and their structures differ in some respects. For example, two C-terminal $\alpha$-helices (residues 381-437), which cover the surface of tubulin and are intimately associated with the globular body of the protein, are not visible in many FtsZ structures from different organisms $^{53-57}$ or are replaced by $\beta$-strands ${ }^{51}$. In addition, the relative positioning of the four subdomains differs in FtsA and $\operatorname{actin}^{58}$, and these two proteins are further distinguished by the unique presence in FtsA of subdomain $1 C^{58}$. Our study extends the similarities between the eukaryotic and prokaryotic cytoskeletons to proteins that contain spectrin-like repeats. An established function of spectrins in eukaryotes is to connect actin filaments to other membrane-associated proteins and, at least in the case of plectin, to also regulate actin dynamics ${ }^{59}$. An analogous role can thus be envisaged for EzrA during cell division, co-ordinating FtsA/FtsZ dynamics inside the cell to peptidoglycan biosynthesis on the outside, an activity that is essential for cytokinesis and which is effected by the recruitment of PBP1 to the site of division by its interaction with $\operatorname{EzrA}^{17}$.

\section{Methods}

Full details of the Methods are supplied in the Supplementary Information.

Expression plasmid construction. The genes encoding fragments of $B s$ and SaEzrA and T7 epitope-tagged FtsZ were cloned into pET-based vectors. The plasmids pBS58 and pCXZ for overexpressing FtsZ were a gift from David Adams (Newcastle University).

Expression and purification of EzrA and EzrA fragments. Recombinant EzrA proteins were produced using E. coli strain BL21(DE3) carrying the appropriate plasmid. Expression was induced in Luria Bertani (LB) medium with isopropyl $\beta$ D-1-thiogalactopyranoside or by auto-induction. For the isopropyl $\beta$-D-1-thiogalactopyranoside -induced expression of selenomethionine-labelled proteins, the methionine-auxotrophic host strain B834 (DE3) was grown in a defined, minimal medium based on M9 supplemented with appropriate ions, vitamins and 19 amino acids; methionine was replaced by selenomethionine. For the BsEzrA proteins, the cells were harvested by centrifugation, before the cell pellet was resuspended in lysis buffer. Cells were lysed by sonication and clarified by centrifugation. The supernatant was filtered and then purified by Ni-nitrilotriacetic acid (Ni-NTA) affinity chromatography with elution effected by imidazole. The hexahistidine tag was removed by overnight incubation with thrombin, before further purification by size exclusion chromatography. The purified proteins were snap-frozen in liquid nitrogen for storage. $\mathrm{SaEzrA}_{24-214}$ was produced without a tag, and was purified by ammonium sulphate fractionation and anion exchange chromatographic steps.

Expression and purification of BsFtsZ. Untagged $B s F t s Z$ was co-expressed with E. coli FtsQ, FtsA and FtsW using plasmids pCXZ and pBS58, as described previously ${ }^{60}$. FtsZ was purified by ion exchange chromatography and serial ammonium sulphate precipitation at 20,30 and $40 \%(\mathrm{w} / \mathrm{v})$. The proteins precipitated with $40 \%$ ammonium sulphate were resuspended and purified further by size exclusion chromatography, before snap-freezing in liquid nitrogen for storage. T7-tagged $B s \mathrm{FtsZ}$ was purified by Ni-NTA affinity chromatography following the same procedures as used for His-tagged BsEzrA fragments, and the His-tag was removed by thrombin.

Crystallization and structure determination. $B s E_{2 r A} A_{22-562}$ was crystallized using a mother liquor of $50 \mathrm{mM}$ MES/NaOH, pH $6.7,1 \mathrm{M}$ tri-sodium citrate, $0.1 \mathrm{M}$ ammonium sulphate, $0.2 \mathrm{M}$ non-detergent sulphobetaine-201. The structure of EzrA was solved by single wavelength anomalous dispersion from a single selenomethionine-labelled crystal. The heavy atoms' substructure and initial phasing were determined in SHELX ${ }^{61}$. SHELXE ${ }^{61}$ and PHASER ${ }^{62}$ were used for subsequent density modification. The atomic model was built by manually docking $\alpha$-helices into the electron density in COOT $^{63}$. The amino-acid sequence was assigned by matching the positions of selenium atoms to methionine positions in the protein sequence. SLOOP ${ }^{64}$ was used to find loops that best fit the electron density map. The geometry of the loop-helix junctions was corrected using RAPPER $^{65}$ and PHENIX. REFINE ${ }^{66}$ was used for refinement of the model.

$\mathrm{SaEzrA} \mathrm{E}_{24-214}$ was crystallized in a buffer of $34 \%$ 2-methyl-2, 4-pentanediol, $0.1 \mathrm{M}$ Tris- $\mathrm{HCl}, \mathrm{pH} 7.5$, and its structure was solved by molecular replacement using equivalent atoms from the $B s E_{22} \mathrm{~A}_{22-52}$ structure as a search model in 
PHASER $^{62}$ and refined to convergence using COOT ${ }^{63}$ and REFMAC5 (ref. 67). Summaries of the data collection and model refinement statistics are provided in Table 1, and Molprobity ${ }^{68}$ was used for structure validation reports.

EDC crosslinking. EzrA fragments were mixed with T7-tagged FtsZ and cross-linked with EDC before analysis by SDS-polyacrylamide gel electrophoresis (SDS-PAGE) and western blotting. To map the crosslink site on EzrA, crosslinked proteins were purified from non-crosslinked proteins by Ni-NTA before NTCB cleavage, SDS-PAGE and immunoblotting with an anti-T7 antibody.

Light scattering. Light scattering measurements used a Varian Cary Eclipse fluorimeter at $30^{\circ} \mathrm{C}$, with all samples in a buffer of $50 \mathrm{mM}$ Tris- $\mathrm{HCl}, \mathrm{pH} 7.3$, $20 \mathrm{mM} \mathrm{KCl}, 3 \mathrm{mM}$ magnesium acetate. Samples were pre-incubated at $30^{\circ} \mathrm{C}$, centrifuged in a microfuge and the supernatant transferred to a quartz microcuvette. GTP was added to a final concentration of $1 \mathrm{mM}$ and light scattering recorded for at least $30 \mathrm{~min}$. The GTP-induced change in light scattering in the presence of EzrA and FtsZ was normalized relative to the average GTP-induced scattering change for FtsZ alone.

Surface plasmon resonance. All SPR experiments used a Biacore X100 instrument with the chip surface at $25^{\circ} \mathrm{C}$. Proteins were immobilized on Biacore CM5 chip surfaces by standard protocols. The binding data were all fit using the Biacore $\mathrm{X}-100$ evaluation software; for affinity fits, the response on the surface $4 \mathrm{~s}$ before the end of the injection were fit as a function of the concentration of the injected EzrA fragment. Each SPR titration shown was performed independently at least twice.

GTPase assay. The GTPase activity of FtsZ was measured under the same buffer conditions as used for the light scattering assays. In an NADH oxidation-coupled continuous regenerative assay ${ }^{69}, 150 \mu \mathrm{l}$ samples were incubated at $30^{\circ} \mathrm{C}$ in a microplate reader after the addition of GTP to $1 \mathrm{mM}$. NADH consumption was measured, which is coupled to GDP production, from the decrease in absorption at $340 \mathrm{~nm}$. For malachite green assays, the release of inorganic phosphate was measured $20 \mathrm{~min}$ after adding GTP to $2 \mathrm{mM}$. A standard curve was generated using phosphate ions in the concentration range $50-450 \mu \mathrm{M}$.

GTP binding. Fluorescence was measured at $22{ }^{\circ} \mathrm{C}$ in a Varian Cary Eclipse fluorimeter. $150 \mu \mathrm{l}$ of $250 \mathrm{nM}$ BODIPY GTP- $\gamma$-S in $50 \mathrm{mM}$ HEPES, $\mathrm{pH} 7,100 \mathrm{mM}$ $\mathrm{KCl}, 5 \mathrm{mM} \mathrm{MgCl} 2$ was preincubated for $>5 \mathrm{~min}$ before adding $4-10 \mu \mathrm{l}$ of a stock solution of either $8 \mu \mathrm{M}$ FtsZ or $8 \mu \mathrm{M}$ FtsZ with $16 \mu \mathrm{M} B s \mathrm{EzrA}_{22-217}$ in the same buffer.

\section{References}

1. Löwe, J. \& Amos, L. Crystal structure of the bacterial cell-division protein FtsZ. Nature 391, 203-206 (1998).

2. Bramhill, D. \& Thompson, C. GTP-dependent polymerization of Escherichia coli FtsZ protein to form tubules. Proc. Natl Acad. Sci. USA 91, 5813-5817 (1994).

3. Adams, D. \& Errington, J. Bacterial cell division: assembly, maintenance and disassembly of the Z ring. Nat. Rev. Microbiol. 7, 642-653 (2009).

4. Bi, E. \& Lutkenhaus, J. FtsZ ring structure associated with division in Escherichia coli. Nature 354, 161-164 (1991).

5. Mukherjee, A. \& Lutkenhaus, J. Dynamic assembly of FtsZ regulated by GTP hydrolysis. EMBO J. 17, 462-469 (1998).

6. Anderson, D., Gueiros-Filho, F. \& Erickson, H. Assembly dynamics of FtsZ rings in Bacillus subtilis and Escherichia coli and effects of FtsZ-regulating proteins. J. Bacteriol. 186, 5775-5781 (2004).

7. Szwedziak, P., Wang, Q., Freund, S. M. \& Löwe, J. FtsA forms actin-like protofilaments. EMBO J. 31, 1-12 (2012).

8. Gamba, P., Veening, J., Saunders, N., Hamoen, L. \& Daniel, R. Two-step assembly dynamics of the Bacillus subtilis divisome. J. Bacteriol. 191, 4186-4194 (2009).

9. Osawa, M., Anderson, D. E. \& Erickson, H. P. Reconstitution of contractile FtsZ rings in liposomes. Science 320, 792-794 (2008).

10. Loose, M. \& Mitchison, T. J. The bacterial cell division proteins FtsA and FtsZ self-organize into dynamic cytoskeletal patterns. Nature Cell Biol. 16, 38-46 (2014).

11. Gueriros-Filho, F. \& Losick, R. A widely conserved bacterial cell division protein that promotes assembly of the tubulin-like protein FtsZ. Genes Dev. 16, 2544-2556 (2002)

12. Gündoğdu, M. et al. Large ring polymers align FtsZ polymers for normal septum formation. EMBO J. 30, 617-626 (2011).

13. Duman, R. et al. Structural and genetic analyses reveal SepF as a new membrane anchor for the Z-ring. Proc. Natl Acad. Sci. USA 110, E4601-E4610 (2013).

14. Hamoen, L. W., Meile, J. C., de Jong, W., Noirot, P. \& Errington, J. SepF, a novel FtsZ-interacting protein required for a late step in cell division. Mol. Microbiol. 59, 989-999 (2006).
15. Levin, P. A., Kurtser, I. G. \& Grossman, A. D. Identification and characterization of a negative regulator of FtsZ ring formation in Bacillus subtilis. Proc. Natl Acad. Sci. USA 96, 9642-9647 (1999).

16. Haeusser, D., Schwartz, R., Smith, A., Oates, M. \& Levin, P. EzrA prevents aberrant cell division by modulating assembly of the cytoskeletal protein FtsZ. Mol. Microbiol. 52, 801-814 (2004).

17. Claessen, D. et al. Control of the cell elongation-division cycle by shuttling of PBP1 protein in Bacillus subtilis. Mol. Microbiol. 68, 1029-1046 (2008).

18. Jorge, A. M., Hoiczyk, E., Gomes, J. P. \& Pinho, M. G. EzrA contributes to the regulation of cell size in Staphylococcus aureus. PLoS ONE 6, e27542 (2011).

19. Chung, K. M., Hsu, H. H., Govindan, S. \& Chang, B. Y. Transcription regulation of ezrA and its effect on cell division of Bacillus subtilis. J. Bacteriol. 186, 5926-5932 (2004).

20. Steele, V. R., Bottomley, A. L., Garcia-Lara, J., Kasturiarachchi, J. \& Foster, S. J. Multiple essential roles for EzrA in cell division of Staphylococcus aureus. Mol. Microbiol. 80, 542-555 (2011).

21. Van Opijnen, T., Bodi, K. \& Camill, A. Tn-seq: high-throughput parallel sequencing for fitness and genetic interaction studies in microorganisms. Nat Methods 6, 767-772 (2009).

22. Thanassi, J. A., Hartman-Neumann, S. L., Dougherty, T. J., Dougherty, B. A. \& Pucci, M. J. Identification of 113 conserved essential genes using a highthroughput gene disruption system in Streptococcus pneumoniae. Nucleic Acids Res. 30, 3152-3162 (2002).

23. Considine, K. M., Sleator, R. D., Kelly, A. L., Fitzgerald, G. F. \& Hill, C. Identification and characterization of an essential gene in Listeria monocytogenes using an inducible gene expression system. Bioeng. Bugs 2, 150-159 (2011)

24. Krissinel, E. \& Henrick, K. Secondary-structure matching (SSM), a new tool for fast protein structure alignment in three dimensions. Acta Crystallogr. D60, 2256-2268 (2004).

25. Holm, L. \& Rosenström, P. Dali server: conservation mapping in 3D. Nucleic Acids Res. 38, W545-W549 (2010).

26. Tung, C. H., Huang, J. W. \& Yang, J. M. Kappa-alpha plot derived structural alphabet and BLOSUM-like substitution matrix for rapid search of protein structure database. Genome Biol. 8, R31 (2007).

27. Borgon, R. A., Vonrhein, C., Bricogne, G., Bois, P. R. \& Izard, T. Crystal structure of human vinculin. Structure 12, 1189-1197 (2004).

28. Liu, J., Taylor, D. W. \& Taylor, K. A. A 3-D reconstruction of smooth muscle alpha-actinin by CryoEM reveals two different conformations at the actinbinding region. J. Mol. Biol. 338, 115-125 (2004).

29. Cansizoglu, A. E., Lee, B. J., Zhang, Z. C., Fontoura, B. M. \& Chook, Y. M. Structure-based design of a pathway-specific nuclear import inhibitor. Nat. Struct. Mol. Biol. 14, 452-454 (2004).

30. Grünwald, M. \& Bono, F. Structure of Importin13-Ubc9 complex: nuclear import and release of a key regulator of sumoylation. EMBO J. 30, 427-438 (2011).

31. Park, S. Y. et al. Reconstruction of the chemotaxis receptor-kinase assembly. Nat. Struct. Mol. Biol. 13, 400-407 (2006).

32. Peter, B. J. et al. BAR domains as sensors of membrane curvature: the amphiphysin BAR structure. Science 495, 495-499 (2004).

33. Bañuelos, S., Saraste, M. \& Djinović Carugo, K. Structural comparisons of calponin homology domains: implications for actin binding. Structure 6, 1419-1431 (1998).

34. Kusunoki, H., MacDonald, R. I. \& Mondragón, A. Structural insights into the stability and flexibility of unusual erythroid spectrin repeats. Structure 12, 645-656 (2004).

35. Stabach, P. R. et al. The structure of the ankyrin-binding site of $\beta$-spectrin reveals how tandem spectrin-repeats generate unique ligand-binding properties. Blood 113, 5377-5384 (2009).

36. Mirijanian, D., Chu, J., Ayton, G. \& Voth, G. Atomistic and coarse-grained analysis of double spectrin repeat units: the molecular origins of flexibility. J. Mol. Biol. 365, 523-534 (2007).

37. Al-Jassar, C. et al. The nonlinear structure of the desmoplakin plakin domain and the effects of cardiomyopathy-linked mutations. J. Mol. Biol. 411, 10491061 (2011).

38. Haeusser, D. P., Garza, A. C., Buscher, A. Z. \& Levin, P. A. The division inhibitor EzrA contains a seven-residue patch required for maintaining the dynamic nature of the medial FtsZ ring. J. Bacteriol. 189, 9001-9010 (2007).

39. Mukherjee, A. \& Lutkenhaus, J. Guanine nucleotide-dependent assembly of FtsZ into filaments. J. Bacteriol. 176, 2754-2758 (1994).

40. Chung, K. M., Hsu, H. H., Yeh, H. Y. \& Chang, B. Y. Mechanism of regulation of prokaryotic tubulin-like GTPase FtsZ by membrane protein EzrA. J. Biol. Chem. 282, 14891-14897 (2007).

41. Buske, P. \& Levin, P. Extreme C-terminus of bacterial cytoskeletal protein FtsZ plays fundamental role in assembly independent of modulatory proteins. J. Biol. Chem. 287, 10945-10957 (2012).

42. Buske, P. \& Levin, P. A flexible C-terminal linker is required for proper FtsZ assembly in vitro and cytokinetic ring formation in vivo. Mol. Microbiol. 89, 249-263 (2013). 
43. Singh, J. K., Makde, R. D., Kumar, V. \& Panda, D. A membrane protein, EzrA, regulates assembly dynamics of FtsZ by interacting with the C-terminal tail of FtsZ. Biochemistry 46, 11013-11022 (2007).

44. Nicolas, A., Delalande, O., Hubert, J. F. \& Le Rumeur, E. The spectrin family of proteins: a unique coiled-coil fold for various molecular surface properties. J. Struct. Biol. 186, 392-401 (2014).

45. Lupas, A. \& Gruber, M. Historical review: another 50th anniversary--new periodicities in coiled coils. Trends Biochem. Sci. 28, 679-685 (2003).

46. Pantazatos, D. P. \& MacDonald, R. I. Site-directed mutagenesis of either the highly conserved Trp- 22 or the moderately conserved Trp-95 to a large, hydrophobic residue reduces the thermodynamic stability of a spectrin repeating unit. J. Biol. Chem. 272, 21052-21059 (1997).

47. MacDonald, R. I., Musacchio, A., Holmgren, R. A. \& Saraste, M. Invariant tryptophan at a shielded site promotes folding of the conformational unit of spectrin. Proc. Natl Acad. Sci. USA 91, 1299-1303 (1994).

48. Pascual, J., Castresana, J. \& Saraste, M. Evolution of the spectrin repeat. Bioessays 19, 811-817 (1997).

49. Grum, V. L., Li, D., MacDonald, R. I. \& Mondragón, A. Structures of two repeats of spectrin suggest models of flexibility. Cell 98, 523-535 (1999).

50. Land, A. D., Luo, Q. \& Levin, P. A. Functional domain analysis of the cell division inhibitor EzrA. PLoS ONE 9, e102616 (2014).

51. Oliva, M., Cordell, S. \& Löwe, J. Structural insights into FtsZ protofilament formation. Nat. Struct. Mol. Biol. 11, 1243-1250 (2004).

52. van den Ent, F., Amos, L. A. \& Löwe, J. Prokaryotic origin of the actin cytoskeleton. Nature 413, 39-44 (2001).

53. van den Ent, F., Amos, L. \& Löwe, J. Bacterial ancestry of actin and tubulin. Curr. Opin. Microbiol. 4, 634-638 (2001).

54. Li, Y. et al. FtsZ protofilaments use a hinge-opening mechanism for constrictive force generation. Science 341, 392-395 (2013).

55. Haydon, D. J. et al. An inhibitor of FtsZ with potent and selective antistaphylococcal activity. Science 321, 1673-1675 (2008).

56. Matsui, T. et al. Structural reorganization of the bacterial cell-division protein FtsZ from Staphylococcus aureus. Acta Crystallogr. D68, 1175-1188 (2012).

57. Oliva, M. A., Trambaiolo, D. \& Löwe, J. Structural insights into the conformational variability of FtsZ. J. Mol. Biol. 373, 1229-1242 (2007).

58. van den Ent, F. \& Löwe, J. Crystal structure of the cell division protein FtsA from Thermotoga maritima. EMBO J. 19, 5300-5307 (2000).

59. Andrä, K., Nikolic, B., Stöcher, M., Drenckhahn, D. \& Wiche, G. Not just scaffolding: plectin regulates actin dynamics in cultured cells. Genes Dev. 12, 3442-3451 (1998).

60. Wang, X. \& Lutkenhaus, J. The FtsZ protein of Bacillus subtilis is localized at the division site and has GTPase activity that is dependent upon FtsZ concentration. Mol. Microbiol. 9, 435-442 (1993).

61. Sheldrick, G. M. A short history of SHELX. Acta Crystallogr. D64, 112-122 (2008).

62. McCoy, A. et al. PHASER crystallographic software. J. Appl. Cryst. 40, 658-674 (2007).

63. Emsley, P., Lohkamp, B., Scott, W. \& Cowtan, K. Features and development of Coot. Acta. Crystallogr. D66, 486-501 (2010).

64. Cowtan, K. Completion of autobuilt protein models using a database of protein fragments. Acta. Crystallogr. D68, 328-335 (2012).

65. Furnham, N. et al. Knowledge-based real-space explorations for low-resolution structure determination. Structure 14, 1313-1320 (2006).
66. Adams, P. et al. PHENIX: a comprehensive Python-based system for macromolecular structure solution. Acta Crystallogr. D66, 213-221 (2010).

67. Murshudov, G. N., Vagin, A. A. \& Dodson, E. J. Refinement of macromolecular structures by the maximum-likelihood method. Acta Crystallogr. D53, 240-255 (1997).

68. Chen, V. B. et al. MolProbity: all-atom structure validation for macromolecular crystallography. Acta Crystallogr. D66, 12-21 (2010).

69. Ingerman, E. \& Nunnari, J. A continuous, regenerative coupled GTPase assay for dynamin-related proteins. Methods Enzymol. 404, 611-619 (2005).

\section{Acknowledgements}

We thank Bert van den Berg, Martin Embley, Jeff Errington, Robert Lightowlers and Tom Williams for useful discussions and comments on the manuscript; David Adams for advice on working with FtsZ; John Berrisford for assistance with low-resolution model building; and Helen Waller, Joseph Newman and Jeremy Lakey for assistance with SPR We also acknowledge the Diamond Light Source and the Australian Synchrotron for access to synchrotron radiation beamlines. SEC-MALLS analyses were undertaken at the University of Manchester Biomolecular Analysis Facility. This work has been supported by the UK BBSRC (BB/G015902/1)

\section{Author contributions}

R.M.C., W.V. and R.J.L. wrote the manuscript; R.M.C. crystallized and solved the BsEzrA structure; R.M.C. and R.J.L. analysed the BsEzrA structure; R.M.C., N.K.B. and L.H. performed biochemical experiments with BsEzrA; A.S. performed sedimentation analyses of BsEzrA; R.A.D. performed two-hybrid analyses; R.M.C., A.S., R.A.D., W.V. and R.J.L. analysed the biochemical and genetic data; J.R.B., Z.-Q.X., N.E.D., E.J.H. and A.J.O. generated the SaEzrA fragments; R.M.C., Z.-Q.X., A.J.O., A.B. and R.J.L. crystallized and solved the $\mathrm{SaEzrA}$ structure; R.M.C., A.J.O., A.B., Z.-Q.X. and R.J.L. analysed the SaEzrA structure and R.M.C., W.V., N.E.D., A.J.O. and R.J.L. revised the manuscript.

\section{Additional information}

Accessions codes. The atomic co-ordinates and the associated structure factors have been deposited in the Protein Data Bank (http://www.pdbe.org) with accession code $4 \mathrm{UXV}$ for $\mathrm{BsEzr}_{\mathrm{A} 22-562}$ and $4 \mathrm{UY} 3$ for $\mathrm{SaEzrA} 4-214$.

Supplementary Information accompanies this paper at http://www.nature.com/ naturecommunications

Competing financial interests: The authors declare no competing financial interests

Reprints and permission information is available online at http://npg.nature.com/ reprintsandpermissions/

How to cite this article: Cleverley, R. M. et al. Structure and function of a spectrin-like regulator of bacterial cytokinesis. Nat. Commun. 5:5421 doi: 10.1038/ncomms6421 (2014)

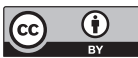

This work is licensed under a Creative Commons Attribution 4.0 International License. The images or other third party material in this article are included in the article's Creative Commons license, unless indicated otherwise in the credit line; if the material is not included under the Creative Commons license, users will need to obtain permission from the license holder to reproduce the material. To view a copy of this license, visit http://creativecommons.org/licenses/by/4.0/ 